\title{
Co-infección por virus del dengue (DEN-V) y virus Chikungunya (CHIK-V) durante un brote epidémico en Cali, Colombia. Comunicación de un caso fatal
}

\author{
Fernando Rosso, Robinson Pacheco, Sarita Rodríguez y Diego Bautista
}

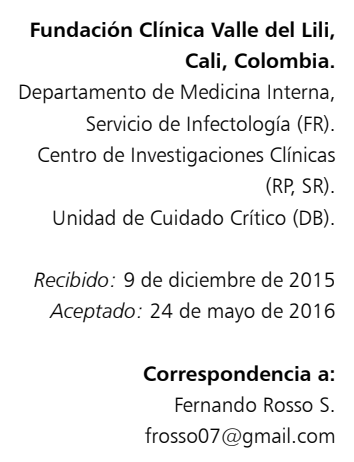

\section{Co-infection by Chikungunya virus (CHIK-V) and dengue virus (DEN-V) during a recent outbreak in Cali, Colombia. Report of a fatal case}

The recent outbreaks of Chikungunya (CHIK-V) virus in endemic areas of dengue (DEN-V) could increase the risk of co-infection. CHIK infection has been considered not severe and with very unusual mortality, however DEN is associated with severe manifestations and increased mortality. Little is known about coinfection. It is possible that co -infection could generate severe cases. We present a case report of co-infection DEN-V -3 and CHIK-V in an elderly patient who developed acute renal failure, dengue shock syndrome (DSS), progresses to multiple organ failure and died. With the recent emergence of CHIK-V in Colombia, the possibility of co-infection with DEN-V should be suspected, especially in severe cases.

Key words: Dengue, Chikungunya fever, coinfection, mortality, shock.

Palabras clave: Dengue, Chikungunya, coinfección, mortalidad, síndrome de choque.

\section{Introducción}

$\mathrm{E}$ 1 virus Chikungunya (CHIK-V), del dengue (DEN-V) y más recientemente el virus Zika, son actualmente los arbovirus de mayor importancia médica. Durante los últimos años, estos virus se han extendido rápidamente por el mundo ${ }^{1-3}$. En la última década, la región de las Américas se ha convertido en un área hiperendémica para DEN-V. Esta situación se agravó desde el año 2014 por la llegada del CHIK-V, que se distribuyó por todo el Caribe, Centro y Sudamérica ${ }^{4-7}$. En Colombia, entre los años 2014 y 2015 , se presentaron alrededor de 438.031 casos presuntivos de Chikungunya.

El valle geográfico del río Cauca, en el suroccidente de Colombia, es una zona hiperendémica con alta transmisión del DEN-V (20\% del total de casos de Colombia). Durante el período 2014 y 2015 se presentaron en la región alrededor de 122.857 casos presuntivos de Chikungunya ${ }^{8}$.

Ambas infecciones (CHIK-V y DEN-V) comparten algunas manifestaciones clínicas, como la fiebre, calofríos, náuseas, vómitos, cefalea, y erupciones cutáneas. En una $\mathrm{u}$ otra infección pueden presentarse dolores articulares y dificultad para la movilización, de intensidad variable. La infección por CHIK-V se ha asociado a mayor compromiso articular ${ }^{9-10}$. Sin embargo, no se ha reportado casos de aumento de la permeabilidad capilar, síndromes de choque o hemorragias graves. En forma infrecuente se han descrito casos fatales con cuadros de encefalopatía, miocarditis y compromiso hepático ${ }^{9,11}$. El DEN-V, por el contrario, se asocia con cuadros clínicos graves, tales como el síndrome de fuga capilar y choque, manifestaciones hemorrágicas, compromiso visceral cardíaco y hepático, con mayor mortalidad ${ }^{12,13}$.

Dado que los vectores son los mismos para ambos arbovirus (mosquitos Aedes aegypti y albopictus) la probabilidad biológica de una co-infección es real. Desde hace varios años se ha descrito la co-circulación de CHIKV y DEN-V en África y en la India, pero este fenómeno es poco común en el sudeste asiático. Se conoce muy poco sobre las manifestaciones clínicas de la co-infección, y no está claro si cuando ésta se presenta, la gravedad del cuadro clínico es mayor ${ }^{11,14}$. La falta de disponibilidad de pruebas diagnósticas de uso clínico rutinario en las áreas endémicas hace difícil la diferenciación de las dos infecciones y, aún más, de la co-infección ${ }^{10,11,15}$.

Presentamos el caso clínico de una infección simultánea por CHIK-V y DEN-V en un adulto mayor que progresó a una falla renal, choque, disfunción orgánica múltiple y muerte.

\section{Caso clínico}

Varón de 72 años, residente del municipio de Miranda (a $50 \mathrm{~km}$ de Cali, Colombia), sin antecedentes mórbidos, consultó a una institución de salud local por un cuadro de seis días de fiebre no cuantificada, poliartralgias, astenia, adinamia y postración. Refería dolor intenso y edema en 
muñecas y tobillos que le limitaban la movilización. Por tener nexo epidemiológico con casos similares se sugirió el diagnóstico de infección por CHIK-V. El paciente fue manejado en forma ambulatoria con anti-inflamatorios no esteroides. Por progresión de los síntomas, y aparición de vómitos y dolor hipogástrico secundario a una retención urinaria, consultó nuevamente dos días después en un centro de primer nivel de atención, donde se manejó con cateterismo vesical. Los exámenes de laboratorio mostraron una leucocitosis de 55.800 céls $/ \mathrm{mm}^{3}$, neutrófilos 49.000 céls $/ \mathrm{mm}^{3}$, linfocitos 3.300 céls $/ \mathrm{mm}^{3}$, plaquetas 160.000 céls $/ \mathrm{mm}^{3}$, hemoglobina $21 \mathrm{~g} / \mathrm{dL}$, hematocrito $57,8 \%$. El examen de orina fue normal. Se derivó a nuestra institución, a los 8 días de evolución, por rápida descompensación hemodinámica con hipotensión y alteración del estado de conciencia. Al examen físico destacaba un edema duro en tobillos. Se encontraba hipo-perfundido, con respiración agónica, presión arterial 86/41 y media de $56 \mathrm{mmHg}$, frecuencia cardíaca 96 por min, frecuencia respiratoria de 26 por min, saturación de oxígeno $84 \%$, $\mathrm{T}^{\circ}$ axilar de $39^{\circ} \mathrm{C}$, frialdad distal, palidez generalizada, llenado capilar mayor de $2 \mathrm{seg}$, ruidos respiratorios disminuidos con roncus generalizados, y abdomen distendido, sin hepatomegalia. En el registro electrocardiográfico se identificaron ondas T picudas sugerentes de hiperkalemia. En la Unidad de Cuidados Intensivos (UCI) se continuó con la reanimación enérgica con fluidos intravenosos. Se evidenció una falla renal (creatinina $3,3 \mathrm{mg} / \mathrm{dL}$ ), sodio $131 \mathrm{meq} / \mathrm{L}$, hiperkalemia de $6 \mathrm{meq} / \mathrm{dl}$, ácido úrico 17 $\mathrm{mg} / \mathrm{dL}$ y albúmina $3 \mathrm{~g} / \mathrm{dL}$. El hemograma evolucionó con leucocitos de 36.400 céls $/ \mathrm{mm}^{3}$, neutrófilos $90 \%$, hemoglobina $22,1 \mathrm{~g} / \mathrm{dL}$, hematocrito $62,5 \%$, recuento de plaquetas 120.000 céls $/ \mathrm{mm}^{3}$ y troponina de 0,45 . En la radiografía de tórax inicial se evidenció un aumento de la trama vascular y elongación del cayado aórtico, sin evidencia de consolidación pulmonar ni derrame pleural. El tiempo parcial de tromboplastina (TPT) inicial fue 46,8 seg y el INR de 0,99. En las siguientes horas, el paciente presentó un deterioro clínico progresivo, con signos de falla respiratoria, por lo que requirió intubación oro-traqueal y ventilación mecánica. A pesar del manejo instaurado el paciente persistió grave, con hemodinamia inestable. Persistió con fiebre hasta $39,7{ }^{\circ} \mathrm{C}$ y acidosis metabólica (Tabla 1). Con diagnóstico de choque secundario con foco desconocido, se inició manejo con fármacos vasopresores, ventilación mecánica invasora, antimicrobianos de amplio espectro y hemofiltración. Al segundo día el paciente continuó en franco deterioro clínico. Un ecocardiograma transtorácico mostró una fracción de eyección conservada, sin otros hallazgos de importancia; la ecografía de abdomen mostró un hígado graso y calcificaciones lineales en riñón derecho. A pesar de la terapia dialítica, el paciente presentó progresión de su falla renal, con acidosis metabólica grave, ácido
Tabla 1. Resultados de laboratorio de un paciente con coinfección por DEN-V y CHIK-V

\begin{tabular}{|c|c|c|c|c|}
\hline Fecha & Día 0 & Día 1 & Día 2 & Día 3 \\
\hline Leucocitos $\times 10^{\wedge} 3 / \mathrm{uL}$ & 36,4 & 36,08 & 37,46 & 42,87 \\
\hline Neutrófilos $\times 10^{\wedge} 3 / \mathrm{uL}$ & 32,7 & 33,2 & 34,46 & 38,92 \\
\hline Linfocitos $\times 10^{\wedge} 3 / \mathrm{uL}$ & 0,728 & 1,08 & 0,746 & 1,98 \\
\hline Plaquetas $\times 10^{\wedge} 3 / \mathrm{uL}$ & 120 & 87 & 43 & 89 \\
\hline Creatinina (mg/dL) & 3,33 & 2,71 & 2,32 & 1,48 \\
\hline Nitrógeno ureico (mg/dL) & 113,20 & 77,10 & 53,00 & 33,40 \\
\hline AST (U/L) & 231,90 & 151,70 & 669,30 & - \\
\hline ALT (U/L) & 78,60 & 45,60 & 185,00 & - \\
\hline Fosfatasa alcalina (U/L) & - & - & 77,00 & - \\
\hline Ácido láctico (mmol/L) & - & 3,11 & 12,63 & 13,61 \\
\hline $\mathrm{LDH}(\mathrm{U} / \mathrm{L})$ & $2.081,00$ & $1.313,00$ & $1.795,00$ & - \\
\hline Ácido úrico (mg/dL) & 17,0 & 9,9 & 4,2 & - \\
\hline Albúmina $(\mathrm{g} / \mathrm{dL})$ & 3,02 & 2,93 & - & - \\
\hline Proteína C Reactiva (mg/dL) & 20,36 & 7,89 & 4,59 & 4,41 \\
\hline $\mathrm{CPK}(\mathrm{U} / \mathrm{L})$ & - & $1.826,00$ & 5.599 & - \\
\hline Potasio (mEq/L) & 6,07 & 5,32 & 5,85 & 6,44 \\
\hline Bilirrubina total (mg/dL) & 1,69 & 1,45 & 2,23 & \\
\hline Bilirrubina directa (mg/dL) & 1,36 & 1,12 & 1,59 & \\
\hline Bilirrubina indirecta $(\mathrm{mg} / \mathrm{dL})$ & 0,33 & 0,33 & 0,64 & \\
\hline
\end{tabular}

láctico muy elevado $(13,6 \mathrm{mmol} / \mathrm{L})$, y una coagulopatía de consumo grave. Presentó un descenso en los valores de hemoglobina hasta $4,1 \mathrm{~g} / \mathrm{dL}$, por lo cual requirió transfusiones de productos sanguíneos. El paciente finalmente falleció al tercer día (60 h) de su ingreso en la institución.

\section{Exámenes microbiológicos}

Se realizó una carga viral por TR-RPC para el CHIK-V la que resultó con 5.000.000 copias/mL de material viral de (LightMix ${ }^{\circledR}$ kit Chikungunya-virus, Light Cycler ${ }^{\circledR}$ de Roche Diagnostics). Después del fallecimiento se recibieron los resultados de TR-RPC positiva para el serotipo DENV-3 realizados en el Laboratorio de Microbiología, Universidad del Valle (Laboratorio autorizado y certificado por el Instituto Nacional de Salud de Colombia, Centro de Referencia Nacional) y la IgM de control anti dengue positiva, mientras que la Ig G y el antígeno NS1 fueron negativos. De un urocultivo, tomado a través del catéter vesical, se aisló Enterococcus faecalis sensible a ampicilina. Además se realizaron dos hemocultivos, uno de los cuales fue positivo para Staphylococcus epidermidis a las $37 \mathrm{~h}$ que se interpretó como contaminación. Las pruebas de IgM para leptospira, la gota gruesa para malaria, y la serología para VIH fueron negativas. Dado que en el sur occidente de Colombia no hay reportes de 
circulación del virus de la fiebre amarilla, no se incluyó en el estudio virológico.

\section{Discusión}

Se ha descrito la co-circulación del CHIKV y el DENV en la India, Malasia y Sri Lanka ${ }^{9,11}$. La co-infección entre estos dos virus se reportó en 1967 en India ${ }^{16}$. Estudios serológicos por anticuerpos IgM positivos para DEN-V y CHIK-V muestran que la co-infección podría presentarse entre 2,7 a $12,4 \%$ de pacientes con un síndrome febril agudo en India ${ }^{17-18}$, aunque está presente la posibilidad de reacciones cruzadas de las pruebas serológicas. En un estudio en el oeste de Bengala (India), se encontró que los pacientes con co-infección se presentaba más en pacientes adultos, tenían menos artritis, y con más diarrea. En todos se evidenció una fiebre bifásica; sin embargo, no reportaron casos fatales ${ }^{9}$.

En 2009, Chahar y cols., comunicaron seis casos de coinfección durante un brote de dengue en Delhi ocurrido en 2006. Todos los pacientes tuvieron fiebre, cefalea, dolor articular y trombocitopenia $<100.000$ céls $/ \mathrm{mm}^{3}$. De estos, dos presentaron fiebre hemorrágica con compromiso del sistema nervioso central y uno de ellos falleció ${ }^{11}$.

Hasta donde tenemos información, este es primer caso fatal de co-infección de CHIK-V y DEN-V descrito en la región de las Américas. A pesar de los hallazgos microbiológicos del paciente (urocultivo positivo a E. faecalis y un hemocultivo a $S$. epidermidis interpretado como contaminante), consideramos que la gravedad y desenlace del cuadro clínico se debió a la co-infección viral.

Se desconoce si la infección por CHIK-V puede exacerbar las manifestaciones de dengue. Algunas series de casos de co-infección han descrito que las complicaciones más frecuentes son las manifestaciones hemorrágicas con compromiso del sistema nervioso central; tampoco se conoce la fisiopatología de la co-infección ni los determinantes de gravedad ${ }^{11}$.

En nuestro caso clínico, el paciente presentó síntomas inespecíficos, característicos de ambas entidades como fiebre, poliartralgias, astenia y malestar general, además de una rápida evolución de los síntomas y el compromiso clínico sistémico, a pesar del manejo y soporte intensivo. Nuestro paciente presentó hemoconcentración y dolor abdominal, lo que sugería signos de alarma de dengue. Con respecto a los exámenes de laboratorio, destaca la marcada leucocitosis con neutrofilia y linfocitosis, que podría asociarse a un desenlace fatal, como lo reportó Harris y cols. en 2003, al igual que la hemoconcentración descrito también como indicador pronóstico ${ }^{19-22}$. Llama la atención que se trató de una infección primaria por dengue (por anticuerpos) y que el paciente desarrollara un curso clínico grave y fatal. En contraste, Schilling comunicó el caso de una paciente con una infección simultánea, quien a pesar de cursar con una infección secundaria grave por dengue no presentó un desenlace fatal ${ }^{23}$.

La presentación clínica de Chikungunya y dengue es más grave en los extremos de la vida ${ }^{24-26}$. Taraphdar y cols., publicaron una serie de casos en que el grupo etario entre los 31 y 40 años fue el más vulnerable a esta co-infección, sin reportar casos con curso grave o fatal. En otra serie clínica de infección simultánea de DEN-V y CHIK-V en 42 pacientes, sólo tres presentaron un curso grave, sin mortalidad ${ }^{27}$. Se ha descrito que la presencia de enfermedades asociadas determina el curso de la infección por DEN-V y CHIK-V ${ }^{9-14}$. En nuestro paciente no se evidenciaron co-morbilidades, salvo su edad avanzada. Lamentablemente no fue posible obtener muestras de tejido ni autorización para realizar un estudio post-mortem.

Factores propios del virus y su interacción con el sistema inmune del hospedero juegan un rol importante en la aparición de casos graves ${ }^{28}$. La diversidad genética entre las cepas virales pudieran explicar en parte las diferencias en su virulencia ${ }^{29}$.

Dado que CHIK-V y DEN-V comparten vectores y zonas geográficas, y pueden cursar con manifestaciones clínicas similares, se debe considerar la co-infección en aquellos pacientes procedentes de áreas endémicas. Es posible que la doble infección pueda producir manifestaciones más graves, en especial en pacientes adultos mayores.

Aunque un paciente puede presentar co-infección por CHIK-V y DEN-V, es difícil determinar el peso de cada infección por separado en la ocurrencia del desenlace.

Se requieren más estudios para evaluar el comportamiento de la co-infección en las Américas.

Agradecimientos. Al cuerpo médico, al Laboratorio clínico y al Departamento de Estadística de la Fundación Clínica Valle del Lili, Cali, Colombia.

\section{Resumen}

La aparición reciente del virus Chikungunya (CHIK-V) en áreas endémicas de dengue (DEN-V) podría aumentar el riesgo de co-infección. Generalmente, se considera que la infección por CHIK-V es de menor gravedad y mortalidad que DEN-V. Poco se conoce sobre la co-infección de DEN-V y CHIK-V, sin embargo, se ha planteado que la co-infección podría ser de mayor gravedad. Se presenta un caso clínico de co-infección por DENV-3 y CHIK-V en un adulto mayor quien desarrolló falla renal aguda, síndrome de choque del dengue, progresión a disfunción orgánica múltiple y muerte. Con la reciente emergencia de CHIK-V en Colombia, es necesario estar atentos ante la posibilidad de co-infección con DEN-V, en especial en casos graves. 


\section{Referencias bibliográficas}

1.- Weaver S C, Forrester N L. Chikungunya: Evolutionary history and recent epidemic spread. Antiviral Res 2015; 120: 32-9.

2.- World Health Organization. Dengue: guidelines for diagnosis, treatment, prevention, and control. New edition. Geneva: World Health Organization; 2009.http://www.who.int/tdr/ publications/documents/dengue-diagnosis.pdf

3.- Roth A, Mercier A, Lepers C, Hoy D, Duituturaga S, Benyon E, et al. Concurrent outbreaks of dengue, chikungunya and Zika virus infections -an unprecedented epidemic wave of mosquito- borne viruses in the Pacific 2012-2014. Euro Surveill 2014; 19: 1-8.

4.- Staples J E, Breiman R F, Powers A M. Chikungunya fever: an epidemiological review of a re-emerging infectious disease. Clin Infect Dis 2009; 49: 942-8.

5.- Instituto Nacional de Salud. Boletín epidemiológico semanal. Semana epidemiológica número 53 - 2014 [acceso: 4 de marzo de 2015]. Disponible en: http:// www.ins.gov.co/boletinepidemiologico/ Boletn\%20Epidemiolgico/2014\%20Boletin \%20 epidemiologico\%20semana\%2053.pdf

6.- Vasconcelos P F, Calisher C H. Emergence of human arboviral diseases in the Americas, 2000-2016. Vector Borne Zoonotic Dis 2016; 16: 295-301.

7.- Rodríguez H, de la Hoz F. Dengue and dengue and vector behaviour in Cáqueza, Colombia, 2004. Rev Salud Pública (Bogotá) 2005; 7 : 1-15.

8.- Instituto Nacional de Salud. Boletín Epidemiológico Semanal. Semana epidemiológica Número 52 - 2015 [acceso: 4 de marzo de 2016). Disponible en: http:// www.ins.gov.co/boletin-epidemiologico/Boletn Epidemiologico/2015 Boletinepidemiologico Semana 52.pdf

9.- Taraphdar D, Sarkar A, Mukhopadhyay B B, Chatterjee S. A comparative study of clinical features between monotypic and dual infection cases with Chikungunya virus and dengue virus in West Bengal, India. Am J Trop Med Hyg 2012; 86: 720-3.
10.- Lee V J, Chow A, Zheng X, Carrasco L R, Cook A R, Lye D C, et al. Simple clinical and laboratory predictors of Chikungunya versus dengue infections in adults. PLoS Negl Trop Dis 2012; 6: e1786.

11.- Chahar H S, Bharaj P, Dar L, Guleria R, Kabra S K, Broor S. Co-infections with Chikungunya virus and dengue virus in Delhi, India. Emerg Infect Dis 2009; 15: 1077-80.

12.- Gubler D J. Dengue and dengue hemorrhagic fever. Clin Microbiol Rev 1998; 11: 480-96.

13.- Hertz J T, Munishi O M, Ooi E E, Howe S, Lim W Y, Chow A, et al. Chikungunya and dengue fever among hospitalized febrile patients in Northern Tanzania. Am J Trop Med Hyg 2012; 86: 171-7.

14.- Borgherini G, Poubeau P, Staikowsky F, Lory M, Le Moullec N, Becquart J P, et al. Outbreak of Chikungunya on Reunion Island: early clinical and laboratory features in 157 adult patients. Clin Infect Dis 2007; 44: 1401-7.

15.- Barde P V, Shukla M K, Bharti P K, Kori B K, Jatav J K, Singh N. Co-circulation of dengue virus serotypes with Chikungunya virus in Madhya Pradesh, central India. WHO South East Asia J Public Health 2014; 3: 36-40. http:// www.searo.who.int/publications/journals/seajph/ seajphv3n1p36.pdf

16.- Chang S F, Su C L, Shu P Y, Yang C F, Liao T L, Cheng C H, et al. Concurrent isolation of chikungunya virus and dengue virus from a patient with coinfection resulting from a trip to Singapore. J Clin Microbiol 2010; 48: 4586-9.

17.- Kalawat U, Sharma K K, Reddy S G. Prevalence of dengue and chickungunya fever and their co-infection. Indian J Pathol Microbiol 2011; 54: 844-6.

18.- Shaikh N, Raut C G Manjunatha M. Coinfections with chikungunya and dengue viruses: a serological study in Karnataka State, India. Indian J Med Microbiol 2015; 33: 459-60.

19.- Harris E, Pérez L, Phares C R, Pérez M de L, Idiaquez W, Rocha J, et al. Fluid intake and decreased risk for hospitalization for dengue fever, Nicaragua. Emerg Infect Dis 2003; 9: 1003-6.
20.- Martínez Torres E. The viruses of dengue and the host's response. Estudos Avançados 2008; 22: 33-52. http://www.scielo.br/pdf/ea/v22n64/ en_a04v2264.pdf

21.- Mena-Castro E, Peña Ch, Pérez-Then E. Dengue en la Clínica Infantil Dr. Robert Reid Cabral. Brote epidémico del año 2006, una oportunidad para identificar parámetros pronósticos. Arch Dom Ped 2008; 41: 7-11.

22.- Díaz A, Kourí G, Guzmán M G, Lobaina L, Bravo J, Ruiz A, et al. Cuadro clínico de la fiebre hemorrágica del dengue/síndrome de choque del dengue en el adulto. Bol Sanit Panam 1988; 104: 560-71. http://iris.paho.org/ xmlui/bitstream/handle/123456789/17650/ v104n6p560.pdf?sequence $=1 \&$ is Allowed=y

23.- Schilling S, Emmerich P, Günther S, SchmidtChanasit J. Dengue and Chikungunya virus co-infection in a German traveller. J Clin Virol 2009; 45: 163-4.

24.- Economopouloua A, Domínguez M, Helynck B, Sissoko D, Wichmann O, Quenel P, et al. Atypical Chikungunya virus infections: clinical manifestations, mortality and risk factors for severe disease during the 2005-2006 outbreak on Reunion. Epidemiol Infect 2009; 137: 53441.

25.- Rajapakse S, Rodrigo C, Rajapakse A. Atypical manifestations of chikungunya infection. Trans R Soc Trop Med Hyg 2010; 104: 89-96.

26.- García-Rivera E J, Rigau-Pérez J G. Dengue severity in the elderly in Puerto Rico. Rev Panam Salud Publica 2003; 13: 362-8.

27.- Omarjee R, Prat C, Flusin O, Boucau S, Tenebray B, Merle O, et al. Importance of case definition to monitor ongoing outbreak of chikungunya virus on a background of actively circulating dengue virus, St Martin, December 2013 to January 2014. Euro Surveill 2014; 19 : 1-3.

28.- Prommalikit O, Thisyakorn U. Dengue virus virulence and diseases severity. Southeast Asian J Trop Med Public Health 2015; 46 Suppl 1: 35-42.

29.- Singh R K, Tiwari S, Mishra V K, Tiwari R, Dhole T N. Molecular epidemiology of Chikungunya virus: mutation in E1 gene region. J Virol Methods 2012; 185: 213-20. 